\title{
Quantitative trait locus analysis of cucumber fruit texture using double-digest restriction-site-associated DNA sequencing
}

Koichiro Shimomura ( $\sim$ shimomur@affrc.go.jp )

National Agriculture and Food Research Organization https://orcid.org/0000-0003-0408-956X

Mitsuhiro Sugiyama

National Agriculture and Food Research Organization

Yoichi Kawazu

National Agriculture and Food Research Organization

Yosuke Yoshioka

University of Tsukuba

\section{Research Article}

Keywords: Cucumber, Firmness, Crispness, Quantitative trait locus, ddRAD-Seq

Posted Date: March 1st, 2021

DOI: https://doi.org/10.21203/rs.3.rs-167431/v1

License: () (i) This work is licensed under a Creative Commons Attribution 4.0 International License. Read Full License 


\section{Abstract}

Fruit firmness and crispness are important traits of cucumber because they directly affect its commercial value. We performed quantitative trait locus (QTL) analysis of these fruit texture traits with the double-digest restriction-site-associated DNA sequencing (ddRAD-Seq) technique and detected 31 QTLs for fruit firmness: 11 for placenta firmness, 12 for skin firmness, and 8 for flesh firmness; and 25 QTLs for crispness-related scores: 10 for flesh crispness index, 8 for flesh apparent fractal dimension by Richardson plot, and 7 for flesh apparent fractal dimension by box-counting (Kolmogorov's dimension). Several QTLs associated with flesh firmness and crispness are located near regions for fruit length, diameter, and length-to-diameter ratio, and for resistance to powdery mildew and downy mildew, indicating that gene linkage is likely to limit breeding efficiency. Our results will contribute to the development of informative DNA markers closely linked to genes for desirable fruit texture traits that are required for effective selection of new cultivars.

\section{Introduction}

Fruit texture traits, such as firmness and crispness, are one of the most important characteristics in cucumber (Cucumis sativus L.), because these are directly related to consumer preference and demand. Grown and bred around the world, the wide range of cucumber cultivars differ greatly in firmness and crispness. In breeding programs, the genetic structure of the breeding materials and the inheritance of target traits are important for efficient breeding. The genetic and physiological mechanisms of fruit texture traits have been investigated by many researchers (Jones 1954; Goffinet 1977; Peterson et al. 1978; Kanno and Kamimura 1980; Suojala-Ahlfors 2005; Yoshioka et al. 2010; Sakata et al. 2011; Shimomura et al. 2016). Although breeding efforts for many fruit vegetables have focused on improvement in texture, few advances have been made in identification of genetic mechanisms, because aspects of texture other than firmness are difficult to measure quantitatively. Cucumber fruit firmness has been quantified by scientific instruments for many years (Breene et al. 1972; Jeon et al. 1973; Kanno and Kamimura 1978; Thompson et al. 1982). However, firmness is just one component of texture, and other components such as crispness and juiciness are also important. Improvements in computer and instrument performance have allowed researchers to develop new methods for the quantitative evaluation of fruit texture traits by mechanical (Horie et al. 2004; Yoshioka et al. 2009, 2010), acoustic (Sakurai et al. 2005), and biorheological (Dan et al. 2003; Kohyama et al. 2009, 2013) measurements.

Considerable advances in the genetic analysis of cucumber fruit traits have been made possible by the development of molecular techniques, such as genetic linkage mapping using molecular markers and quantitative trait locus (QTL) analysis. Many genetic analyses associated with fruit length, diameter, and length-to-diameter ratio $(L / D)$ have been performed. The first report of $Q T L$ analysis for cucumber morphological traits identified 18 QTLs: 5 for length, 3 for diameter, 3 for seed-cavity size, 2 for color, 4 for L/D, and 1 for seed-cavity-to-diameter ratio (Kennard and Havey 1995). Later researchers reported many QTLs for fruit morphological traits (Serquen et al. 1997; Fazio et al. 2003; Yuan et al. 2008a, b; Bo et al. 2015; Weng et al. 2015; Pan et al. 2017; Shimomura et al. 2017; Gao et al. 2020; Wang et al. 2020a, b). Methods of single nucleotide polymorphism (SNP) genotyping by next-generation sequencing (NGS), such as restriction-site-associated DNA sequencing (RAD-Seq) and genotyping-by-sequencing techniques, have been widely adopted on account of their low cost and flexibility (Davey et al. 2011). In cucumber, NGS investigations include genetic analysis of fruit-related traits. Wei et al. (2014) reported the first QTL analysis using an NGS-derived genetic map in cucumber; using the specific-length amplified fragment sequencing technique they identified 7 QTLs for fruit length and 2 QTLs for fruit weight. However, no QTL analysis has yet been performed for fruit texture traits in cucumber.

Here, we report the first QTL analysis of fruit firmness and crispness, using double-digest RAD-Seq (ddRAD-Seq) analysis. We investigated three populations of $F_{2}$ progeny of parents with high and low crispness: two of Japanese-type cucumber $\times$ weedy cucumber line CS-PMR1, and one of Japanese-type cucumber $\times$ Beit Alpha-type cucumber. CS-PMR1, derived from PI197088, has strong resistance to both powdery mildew and downy mildew, and can be used to breed new cultivars with resistance to these diseases (Fukino et al. 2013; Morishita et al. 2003; Sakata et al. 2006). However, the development of DNA markers closely linked to genes for both disease resistance and fruit texture traits is needed for the efficient breeding of new cultivars. Here, we document the inheritance of fruit texture traits and discuss the prospects for breeding new cucumber cultivars with disease resistance and desirable fruit texture traits.

\section{Materials And Methods}

Page 2/18 


\section{Plant materials}

Phenotypic data on fruit texture traits were collected in 2012, 2014, and 2016 at the Institute of Vegetable and Floriculture Science, National Agriculture and Food Research Organization, Mie, Japan. We used three populations of $F_{2}$ progeny of parents with high and low crispness (Fig. 1). Populations of 116 and $128 F_{2}$ progeny (EC1 and EC2, respectively) were derived from a cross between high-crisp line 'Encore 10' (“E”), an inbred line derived from a commercial $F_{1}$ hybrid cultivar, and low-crisp line CS-PMR1 ("C"; weedytype cucumber). EC1 and EC2 populations were sown in plastic pots on 11 Apr 2012 and 21 Apr 2014 and transplanted into a plastic greenhouse on 2 May 2012 and 9 May 2014. A population of $163 F_{2}$ progeny (EA) was derived from a cross between 'Encore 10' and low-crisp line 'Atar' ("A"; Beit Alpha type), an inbred line derived from a commercial $F_{1}$ hybrid cultivar. The EA population was sown on 25 Apr 2016 and transplanted on 10 May 2016. Plants set fruit 1 month after transplanting. Fruits for puncture tests were harvested on 26 May to 15 June 2012, 11 June to 15 July 2014, and 13 June to 22 July 2016. These fruits weighed around $100 \mathrm{~g}$, the Japanese market standard.

\section{Evaluation of fruit texture}

We evaluated the texture of the sampled fruits by puncture test as described previously (Yoshioka et al. 2009, 2010; Shimomura et al. 2016). A 15-mm-thick transverse slice was cut from the middle of each fruit. The samples were punctured with a Texture Analyzer (Stable Micro Systems Ltd., Surrey, UK) fitted with a 3.0-mm-diameter cylindrical plunger. The rate of advance was $150 \mathrm{~mm}$ $\mathrm{min}^{-1}$, and data were acquired at a resolution of 500 points per second. Transverse sections were punctured in the direction of the long axis of the fruit to a distance of $8 \mathrm{~mm}$ : the placenta was punctured once, and the fleshy part was punctured at three separate locations. The middle two-thirds of each force-deformation curve (puncture depth 2.5 to $7.6 \mathrm{~mm}$ ) was used to calculate placenta firmness ( $\mathrm{PFI}$ ), flesh firmness ( $\mathrm{FFI})$, and three flesh crispness scores: flesh crispness index (FCl) and the apparent fractal dimension of each curve by the Richardson plot (FFDR) and box-counting (Kolmogorov's dimension; FFDK) methods (Russ 1994; Peleg 1997; Roudaut et al. 2002; Horie et al. 2004; Yoshioka et al. 2009). For the evaluation of skin firmness (SFI) with a $30^{\circ}$ wedge plunger, the samples were punctured from outside to inside to a distance of $4 \mathrm{~mm}$ at three locations. The first peak force in each forcedeformation curve was recorded and used as SFI. The data were analyzed in R software v. 4.0.2 (R Development Core Team, 2005).

\section{Double-digest restriction-site-associated DNA sequencing (ddRAD-Seq)}

Library construction and sequencing analysis of the three populations and the parents were performed as described by Shirasawa et al. (2016) with minor modifications. The ddRAD-Seq libraries were constructed with two combinations of restriction enzymes, Pstl and Mspl (Thermo Fisher Scientific, Waltham, MA, USA). Digested DNA was ligated to adapters using T4 DNA ligase (Takara Bio Inc., Shiga, Japan) and purified using Agencourt AMPure XP cleanup reagent (Beckman Coulter, Brea, CA, USA) to eliminate short (< 300 bp) DNA fragments. Purified DNA was amplified by PCR with indexed primers. Amplified DNA fragments were purified using the Qiagen Mini Elute Kit (Qiagen, Hilden, Germany), and fragments 300-1000 bp in length were fractionated by $2.0 \%$ agarose gel electrophoresis (Kanto Chemical Co., Inc., Tokyo, Japan) with a QIAquick Gel Extraction Kit (Qiagen). These libraries were sequenced on a HiSeq X sequencer (Illumina, Inc., San Diego, USA) in 150-bp paired-end reads.

\section{NGS data acquisition and SNP calling}

The sequencing data without low-quality reads were trimmed and filtered in FaQCs v. 2.08 software (Lo and Chain 2014). Then the data were mapped to the cucumber reference genome ('Chinese Long' v3) sequence (Li et al. 2019) in the Cucurbit Genomics Database of the International Cucurbit Genomics Initiative (http://www.icugi.org) in BWA-MEM v. 0.7.17 software (Li 2013). The resulting sequence alignment/map (SAM) format files were converted to binary sequence alignment/map (BAM) format files. SNP calling and genotyping were performed with the mpileup tool in SAMtools v. 0.1.15 and BCFtools v. 1.9 software (Li 2011). SNPs with $>8$ reads in $>80 \%$ of progeny were used for analysis.

\section{SSR markers}

We used simple sequence repeat (SSR) markers from previous studies (Fukino et al. 2008; Ren et al. 2009; Cavagnaro et al. 2010) to test for polymorphism between parents of EC1. PCR amplification was carried out using a post-labeling method for multiplexed genotyping analysis with a bar-coded split tag (BStag) as described by Shimizu and Yano (2011) with minor modifications. PCR was performed in a $10-\mu \mathrm{L}$ reaction mixture that consisted of $1-5 \mathrm{ng}$ of genomic DNA, $0.5 \mathrm{pmol}$ of both the tagged forward primer 
and the fluorescently labeled BStag primer, 2 pmol of reverse primer, and 2× Qiagen Multiplex PCR Master Mix (Qiagen). DNA was amplified in a Mastercycler Pro 384 (Eppendorf Corporation, Hamburg, Germany) under the following protocol: $95^{\circ} \mathrm{C}$ for $5 \mathrm{~min} ; 33$ cycles of $95^{\circ} \mathrm{C}$ for $20 \mathrm{~s}, 55^{\circ} \mathrm{C}$ for $1 \mathrm{~min} 30 \mathrm{~s}, 72{ }^{\circ} \mathrm{C}$ for $30 \mathrm{~s}$; an additional three cycles of $95^{\circ} \mathrm{C}$ for $20 \mathrm{~s}, 49^{\circ} \mathrm{C}$ for $1 \mathrm{~min} 30 \mathrm{~s}, 72{ }^{\circ} \mathrm{C}$ for $30 \mathrm{~s}$; and $68^{\circ} \mathrm{C}$ for $10 \mathrm{~min}$. The sizes of the amplified fragments were estimated on an automated DNA analyzer (model 3730xl, Applied Biosystems, Foster City, CA, USA) with a GeneScan-500LIZ size standard (Applied Biosystems). Fragment length was determined in GeneMapper software (Applied Biosystems).

\section{Linkage map construction and QTL analysis}

Three genetic linkage maps were constructed in AntMap v. 1.2 software (Iwata and Ninomiya 2006) from the SNP markers obtained by ddRAD-Seq analysis. The SNP markers were selected by removing low-quality loci ( $>5$ progeny have missing values at each locus). For EC1, 103 additional SSR markers were used. QTL analysis for each texture trait was performed by composite interval mapping in Windows QTL Cartographer v. 2.5 software (Wang et al. 2007) with the following parameter settings: model 6, forward and backward stepwise regression model, 5 maximum background marker loci, window size 10, and $1 \mathrm{cM}$ walking speed along chromosomes. The logarithm of odds (LOD) thresholds for QTL detection in both mapping methods were determined by 1000 permutations.

\section{Results}

The continuous distributions of fruit texture traits in the three $F_{2}$ populations suggest polygenic control of these traits (Fig. 2). As expected, the high-crisp parent ('Encore 10') had high crispness-related scores (FCl, FFDR, FFDK) and the low-crisp parents (CSPMR1 and 'Atar') had low scores. On the other hand, placenta, skin, and flesh firmness showed different features: both 'Encore 10' and 'Atar' had similar scores for PFI and SFl; and both parents of all populations had similar scores for FFI (Fig. 2; Table S1).

ddRAD-Seq analysis returned an average of 0.5 million high-quality reads per sample with an average Q30 quality score (indicating a $0.1 \%$ chance of error and $99.9 \%$ confidence) of 94.38 at 279 out of 2664 SNP sites in EC1, 1.7 million reads with Q30 $=90.26$ at $607 / 3571$ SNPs in EC2, and 1.3 million reads with Q30 $=94.54$ at 496/2093 SNPs in EA. With the 103 additional SSR markers in EC1, we used 382 (EC1), 607 (EC2), and 496 (EA) markers for constructing linkage maps. Each map has seven linkage groups (LGs), and spans $946.31 \mathrm{cM}$ (with an average distance between markers of $2.48 \mathrm{cM}$ ) in EC1, $661.81 \mathrm{cM}(1.09 \mathrm{cM})$ in EC2, and $706.54 \mathrm{cM}$ $(1.42 \mathrm{cM})$ in EA. All sets of seven LGs correspond to the cucumber 'Chinese Long' v3 reference genome (Li et al. 2019). The order of markers was almost entirely conserved throughout the three maps (Fig. 3). EA had long regions with no markers (>10 cM) of $14.13 \mathrm{cM}$ on Chr. 2, $12.25 \mathrm{cM}$ on Chr. 4, $11.15 \mathrm{cM}$ on Chr. 5, and $16.95 \mathrm{cM}$ on Chr. 6. EC1 had only one large gap, of $10.11 \mathrm{cM}$ on Chr. 5. EC2 had no large gaps.

Many QTLs for fruit texture traits detected were significant at LOD thresholds of 3.5-3.8 (Fig. 4). The LOD peaks of these QTLs ranged from 3.53 to 34.12 , and the proportion of phenotypic variation explained $\left(R^{2}\right)$ ranged from 0.04 to 0.55 (Table 1 ). QTLs for each texture trait were detected in the same or near regions of EC1 and EC2 if regions where their LOD scores exceeded the threshold overlapped. On the other hand, the detection of some QTLs in different regions between EC and EA was considered to be caused by the different parental line combinations. In total, 56 QTLs for texture traits were detected: 11 for PFI, 12 for SFI, 8 for FFI, 10 for FCl, 8 for FFDR, and 7 for FFDK (Fig. 4; Table 1). 
Table 1

QTLs for fruit texture traits detected by composite interval mapping in $\mathrm{F}_{2}$ populations from 'Encore 10' $\times$ CS-PMR1 cross (EC1, EC2) and 'Encore 10' × 'Atar' cross (EA).

\begin{tabular}{|c|c|c|c|c|c|c|c|c|c|c|c|}
\hline Trait & Chr. & $\mathrm{QTL}^{\mathrm{ab}}$ & & $\begin{array}{l}\text { Positior } \\
\text { (cM) }\end{array}$ & & LOD pe & & $\begin{array}{l}\text { Contributing } \\
\text { parent }\end{array}$ & $\begin{array}{l}\text { Additive } \\
\text { effect }\end{array}$ & $\begin{array}{l}\text { Estimated } \\
\text { range } \\
\text { within } \\
\text { 'Chinese } \\
\text { Long' v3 } \\
\text { genome }\end{array}$ & $R^{2 \mathrm{c}}$ \\
\hline \multicolumn{12}{|c|}{$\begin{array}{l}\text { Placenta } \\
\text { firmness (PFI) }\end{array}$} \\
\hline \multirow[t]{5}{*}{ EC1 } & & $\begin{array}{l}\text { pfi- } \\
1.2\end{array}$ & 72.0 & & 3.65 & & $\begin{array}{l}\text { Encore } \\
10\end{array}$ & 0.074 & $\begin{array}{l}17938051- \\
18305320\end{array}$ & 0.07 & \\
\hline & & $\begin{array}{l}p f i- \\
2.1\end{array}$ & 53.4 & & 9.96 & & $\begin{array}{l}\text { Encore } \\
10\end{array}$ & 0.117 & $\begin{array}{l}8268910- \\
15656494\end{array}$ & 0.19 & \\
\hline & 4 & $\begin{array}{l}p f \\
4 .\end{array}$ & & 42.7 & & 3.74 & & Encore 10 & 0.068 & $\begin{array}{l}2350185- \\
5938439\end{array}$ & 0.07 \\
\hline & 6 & $\begin{array}{l}p f \\
6 .\end{array}$ & & 71.6 & & 7.86 & & CS-PMR1 & -0.106 & $\begin{array}{l}10124104- \\
17307123\end{array}$ & 0.15 \\
\hline & 6 & $\begin{array}{l}p f \\
6 .\end{array}$ & & 118.9 & & 7.19 & & Encore 10 & 0.113 & $\begin{array}{l}20945018- \\
26073622\end{array}$ & 0.15 \\
\hline \multirow[t]{5}{*}{ EC2 } & 1 & pf & & 85.4 & & 11.29 & & Encore 10 & 0.278 & $\begin{array}{l}24765386- \\
32324446\end{array}$ & 0.18 \\
\hline & 2 & pf & & 43.8 & & 6.95 & & Encore 10 & 0.241 & $\begin{array}{l}9863544- \\
14476230\end{array}$ & 0.10 \\
\hline & 4 & $\begin{array}{l}p f \\
4 .\end{array}$ & & 56.2 & & 5.99 & & Encore 10 & 0.214 & $\begin{array}{l}9614597- \\
17359638\end{array}$ & 0.09 \\
\hline & 6 & $\begin{array}{l}p f \\
6 .\end{array}$ & & 42.1 & & 5.01 & & CS-PMR1 & -0.192 & $\begin{array}{l}9100225- \\
13529188\end{array}$ & 0.07 \\
\hline & 6 & $\begin{array}{l}p f \\
6 .\end{array}$ & & 68.7 & & 7.62 & & Encore 10 & 0.263 & $\begin{array}{l}21270182- \\
28170725\end{array}$ & 0.11 \\
\hline \multirow[t]{5}{*}{ EA } & 1 & $\begin{array}{l}p f \\
7 .\end{array}$ & & 18.0 & & 8.55 & & Encore 10 & 0.335 & $\begin{array}{l}1172237- \\
6198629\end{array}$ & 0.16 \\
\hline & 2 & pf & & 28.8 & & 5.44 & & Encore 10 & 0.249 & $\begin{array}{l}4695169- \\
12618347\end{array}$ & 0.08 \\
\hline & 2 & pf & & 64.9 & & 4.60 & & Encore 10 & 0.192 & $\begin{array}{l}17757949- \\
20408580\end{array}$ & 0.07 \\
\hline & & $\begin{array}{l}\text { pfi- } \\
3.1\end{array}$ & 35.0 & & 8.66 & & $\begin{array}{l}\text { Encore } \\
10\end{array}$ & 0.317 & $\begin{array}{l}6860994- \\
12201882\end{array}$ & 0.17 & \\
\hline & 4 & $\begin{array}{l}p f \\
4 .\end{array}$ & & 79.4 & & 5.45 & & Atar & -0.217 & $\begin{array}{l}17493968- \\
22922654\end{array}$ & 0.10 \\
\hline $\begin{array}{l}\text { Skin } \\
\text { firmness } \\
\text { (SFI) }\end{array}$ & & & & & & & & & & & \\
\hline
\end{tabular}

a pfi, placenta firmness; sfi, skin firmness; ffi, flesh firmness; $f c i$, flesh crispness index; ffdr, flesh apparent fractal dimension based on Richardson plot; ffdk, flesh apparent fractal dimension based on box-counting (Kolmogorov's dimension).

${ }^{b} s f i-1.2^{E}$ is contributed by 'Encore $10^{\prime}$ inbred line, sfi-3. $7^{A}$ is contributed by 'Atar' inbred line, $s f i-1.2^{C}$ and $s f i-3.1^{C}$ are contributed by CS-PMR1.

${ }^{\mathrm{c}} R^{2}$ is the proportion of phenotypic variance explained by the QTL. 


\begin{tabular}{|c|c|c|c|c|c|c|c|c|}
\hline Trait & Chr. & $\mathrm{QTL}^{\mathrm{ab}}$ & $\begin{array}{l}\text { Position } \\
\text { (cM) }\end{array}$ & LOD peak & $\begin{array}{l}\text { Contributing } \\
\text { parent }\end{array}$ & $\begin{array}{l}\text { Additive } \\
\text { effect }\end{array}$ & $\begin{array}{l}\text { Estimated } \\
\text { range } \\
\text { within } \\
\text { 'Chinese } \\
\text { Long' v3 } \\
\text { genome }\end{array}$ & $R^{2 c}$ \\
\hline \multirow[t]{3}{*}{ EC1 } & 1 & $\begin{array}{l}s f i- \\
1.2^{c}\end{array}$ & 65.7 & 6.47 & CS-PMR1 & -2.260 & $\begin{array}{l}8218171- \\
19041404\end{array}$ & 0.14 \\
\hline & 2 & $\begin{array}{l}\text { sfi- } \\
2.1\end{array}$ & 31.2 & 7.26 & CS-PMR1 & -1.919 & $\begin{array}{l}4201305- \\
10280790\end{array}$ & 0.15 \\
\hline & 3 & $\begin{array}{l}\text { sfi- } \\
3.1^{c}\end{array}$ & 6.0 & 8.09 & CS-PMR1 & -2.609 & $\begin{array}{l}319052- \\
8140626\end{array}$ & 0.19 \\
\hline \multirow[t]{6}{*}{ EC2 } & 1 & $\begin{array}{l}\text { sfi- } \\
1.1\end{array}$ & 5.0 & 7.36 & CS-PMR1 & -2.082 & $\begin{array}{l}62696- \\
3536162\end{array}$ & 0.12 \\
\hline & 1 & $\begin{array}{l}\text { sfi- } \\
1.3\end{array}$ & 68.4 & 6.69 & CS-PMR1 & -2.032 & $\begin{array}{l}19041404- \\
25266488\end{array}$ & 0.12 \\
\hline & 2 & $\begin{array}{l}\text { sfi- } \\
2.1\end{array}$ & 44.2 & 5.02 & CS-PMR1 & -2.146 & $\begin{array}{l}9863544- \\
14206511\end{array}$ & 0.08 \\
\hline & 3 & $\begin{array}{l}\text { sfi- } \\
3.7^{C}\end{array}$ & 3.2 & 9.37 & CS-PMR1 & -2.755 & $\begin{array}{l}352951- \\
6845050\end{array}$ & 0.17 \\
\hline & 3 & $\begin{array}{l}\text { sfi- } \\
3.2\end{array}$ & 66.7 & 4.15 & CS-PMR1 & -1.539 & $\begin{array}{l}29875711- \\
30534604\end{array}$ & 0.06 \\
\hline & 6 & $\begin{array}{l}s f i- \\
6.2\end{array}$ & 78.8 & 3.64 & CS-PMR1 & -1.422 & $\begin{array}{l}24568370- \\
24624709\end{array}$ & 0.06 \\
\hline \multirow[t]{5}{*}{ EA } & 1 & $\begin{array}{l}s f i^{-} \\
1.2^{E}\end{array}$ & 34.7 & 15.49 & Encore 10 & 2.760 & $\begin{array}{l}4246297- \\
10199104\end{array}$ & 0.27 \\
\hline & 3 & $\begin{array}{l}\text { sfi- } \\
3.7^{A}\end{array}$ & 2.2 & 4.56 & Atar & -1.258 & $\begin{array}{l}348913- \\
3132790\end{array}$ & 0.07 \\
\hline & 4 & $\begin{array}{l}\text { sfi- } \\
4.1\end{array}$ & 64.4 & 4.75 & Atar & -1.339 & $\begin{array}{l}15188653- \\
18675455\end{array}$ & 0.08 \\
\hline & 5 & $\begin{array}{l}\text { sfi- } \\
5.1\end{array}$ & 62.3 & 5.16 & Atar & -1.351 & $\begin{array}{l}17381318- \\
25137285\end{array}$ & 0.08 \\
\hline & 6 & $\begin{array}{l}\text { sfi- } \\
6.1\end{array}$ & 57.5 & 7.03 & Encore 10 & 1.221 & $\begin{array}{l}10340732- \\
21093120\end{array}$ & 0.10 \\
\hline \multicolumn{9}{|l|}{$\begin{array}{l}\text { Flesh } \\
\text { firmness } \\
\text { (FFI) }\end{array}$} \\
\hline \multirow[t]{3}{*}{ EC1 } & 1 & $\begin{array}{l}\mathrm{ffi}- \\
1.1\end{array}$ & 17.5 & 5.41 & Encore 10 & 0.300 & $\begin{array}{l}1345396- \\
4183118\end{array}$ & 0.14 \\
\hline & 1 & $\begin{array}{l}\mathrm{ffi}- \\
1.2\end{array}$ & 76.2 & 7.60 & Encore 10 & 0.396 & $\begin{array}{l}4808143- \\
23134200\end{array}$ & 0.20 \\
\hline & 6 & $\begin{array}{l}\mathrm{ffl}- \\
6.3\end{array}$ & 112.5 & 3.93 & Encore 10 & 0.290 & $\begin{array}{l}21213122- \\
23012045\end{array}$ & 0.12 \\
\hline
\end{tabular}

a $p f i$, placenta firmness; sfi, skin firmness; ffi, flesh firmness; fci, flesh crispness index; ffdr, flesh apparent fractal dimension based on Richardson plot; ffdk, flesh apparent fractal dimension based on box-counting (Kolmogorov's dimension).

${ }^{b} s f i-1.2^{E}$ is contributed by 'Encore $10^{\prime}$ inbred line, sfi-3. $1^{A}$ is contributed by 'Atar' inbred line, sfi- $1.2^{C}$ and $s f i-3.1^{C}$ are contributed by CS-PMR1.

${ }^{\mathrm{c}} R^{2}$ is the proportion of phenotypic variance explained by the QTL. 


\begin{tabular}{|c|c|c|c|c|c|c|c|c|}
\hline Trait & Chr. & $\mathrm{QTL}^{\mathrm{ab}}$ & $\begin{array}{l}\text { Position } \\
\text { (cM) }\end{array}$ & LOD peak & $\begin{array}{l}\text { Contributing } \\
\text { parent }\end{array}$ & $\begin{array}{l}\text { Additive } \\
\text { effect }\end{array}$ & $\begin{array}{l}\text { Estimated } \\
\text { range } \\
\text { within } \\
\text { 'Chinese } \\
\text { Long' v3 } \\
\text { genome }\end{array}$ & $R^{2 c}$ \\
\hline \multirow[t]{3}{*}{ EC2 } & 1 & $\begin{array}{l}f f i- \\
1.2\end{array}$ & 54.2 & 10.18 & Encore 10 & 0.447 & $\begin{array}{l}10460732- \\
21590246\end{array}$ & 0.25 \\
\hline & 6 & $\begin{array}{l}\mathrm{ffi}- \\
6.1\end{array}$ & 32.9 & 5.57 & CS-PMR1 & -0.236 & $\begin{array}{l}6089049- \\
10783025\end{array}$ & 0.12 \\
\hline & 6 & $\begin{array}{l}\mathrm{ffi}- \\
6.4\end{array}$ & 92.0 & 5.31 & Encore 10 & 0.262 & $\begin{array}{l}28218841- \\
30759549\end{array}$ & 0.11 \\
\hline \multirow[t]{4}{*}{ EA } & 1 & $\begin{array}{l}\mathrm{ffi}- \\
1.1\end{array}$ & 16.6 & 34.12 & Encore 10 & 0.609 & $\begin{array}{l}1779920- \\
6198629\end{array}$ & 0.55 \\
\hline & 3 & $\begin{array}{l}f f i- \\
3.1\end{array}$ & 26.8 & 4.41 & Encore 10 & 0.179 & $\begin{array}{l}6229032- \\
11661083\end{array}$ & 0.05 \\
\hline & 4 & $\begin{array}{l}\mathrm{ffi}- \\
4.1\end{array}$ & 71.4 & 4.04 & Atar & -0.123 & $\begin{array}{l}17493968- \\
17974470\end{array}$ & 0.04 \\
\hline & 6 & $\begin{array}{l}\mathrm{ffi}- \\
6.2\end{array}$ & 57.5 & 6.72 & Encore 10 & 0.159 & $\begin{array}{l}13642268- \\
20075212\end{array}$ & 0.07 \\
\hline \multicolumn{9}{|c|}{$\begin{array}{l}\text { a pfi, placenta firmness; sfi, skin firmness; ffi, flesh firmness; fci, flesh crispness index; ffdr, flesh apparent fractal dimension } \\
\text { based on Richardson plot; ffdk, flesh apparent fractal dimension based on box-counting (Kolmogorov's dimension). }\end{array}$} \\
\hline \multicolumn{9}{|c|}{$\begin{array}{l}\text { b } s f i-1.2^{E} \text { is contributed by 'Encore } 10^{\prime} \text { inbred line, sfi-3. } 1^{A} \text { is contributed by 'Atar' inbred line, sfi- } 1.2^{C} \text { and } s f i-3.1^{C} \text { are contributed } \\
\text { by CS-PMR1. }\end{array}$} \\
\hline
\end{tabular}


Table 1

(Continued).

\begin{tabular}{|c|c|c|c|c|c|c|c|c|}
\hline Trait & Chr. & $\mathrm{QTL}^{\mathrm{ab}}$ & $\begin{array}{l}\text { Position } \\
\text { (cM) }\end{array}$ & LOD peak & $\begin{array}{l}\text { Contributing } \\
\text { parent }\end{array}$ & $\begin{array}{l}\text { Additive } \\
\text { effect }\end{array}$ & $\begin{array}{l}\text { Estimated range within } \\
\text { 'Chinese Long' v3 genome }\end{array}$ & $R^{2 c}$ \\
\hline \multicolumn{9}{|c|}{$\begin{array}{l}\text { Flesh crispness } \\
\text { index (FCl) }\end{array}$} \\
\hline EC1 & 1 & $\begin{array}{l}f c i- \\
1.1\end{array}$ & 5.2 & 9.77 & Encore 10 & 0.109 & $77153-2993540$ & 0.20 \\
\hline & 3 & $\begin{array}{l}f c i- \\
3.1\end{array}$ & 81.8 & 9.94 & Encore 10 & 0.117 & $16211979-28040750$ & 0.23 \\
\hline & 5 & $\begin{array}{l}f c i- \\
5.1\end{array}$ & 38.3 & 4.32 & Encore 10 & 0.071 & $5591997-6531518$ & 0.09 \\
\hline & 6 & $\begin{array}{l}f c i- \\
6.1\end{array}$ & 95.4 & 5.19 & Encore 10 & 0.018 & $18728426-21213122$ & 0.09 \\
\hline EC2 & 1 & $\begin{array}{l}f c i- \\
1.1\end{array}$ & 14.8 & 17.61 & Encore 10 & 0.193 & $62696-5485409$ & 0.28 \\
\hline & 1 & $\begin{array}{l}f c i- \\
1.2\end{array}$ & 81.2 & 4.20 & Encore 10 & 0.037 & $25350961-26438849$ & 0.05 \\
\hline & 3 & $\begin{array}{l}f_{c i}- \\
3.1\end{array}$ & 48.1 & 4.06 & Encore 10 & 0.105 & $17838480-20917609$ & 0.05 \\
\hline & 4 & $\begin{array}{l}f c i- \\
4.1\end{array}$ & 72.6 & 8.43 & CS-PMR1 & -0.139 & $17623371-23097150$ & 0.13 \\
\hline & 6 & $\begin{array}{l}f c i- \\
6.3\end{array}$ & 90.8 & 4.05 & Encore 10 & 0.080 & $28170725-29384179$ & 0.05 \\
\hline EA & 1 & $\begin{array}{l}f c i- \\
1.1\end{array}$ & 1.0 & 3.74 & Encore 10 & 0.081 & $198244-989498$ & 0.06 \\
\hline & 2 & $\begin{array}{l}f c i- \\
2.1\end{array}$ & 59.2 & 6.67 & Atar & -0.102 & $15568875-20408580$ & 0.11 \\
\hline & 3 & $\begin{array}{l}f c i- \\
3.1\end{array}$ & 70.2 & 7.86 & Encore 10 & 0.118 & $19323744-29655770$ & 0.13 \\
\hline & 5 & $\begin{array}{l}f c i- \\
5.2\end{array}$ & 66.9 & 4.04 & Encore 10 & 0.080 & $22358151-24753912$ & 0.07 \\
\hline & 6 & $\begin{array}{l}f c i- \\
6.1\end{array}$ & 61.7 & 14.50 & Encore 10 & 0.135 & $10340732-21093120$ & 0.24 \\
\hline & 6 & $\begin{array}{l}f c i- \\
6.2\end{array}$ & 90.3 & 3.64 & Encore 10 & 0.061 & $24693006-26147197$ & 0.05 \\
\hline \multicolumn{9}{|c|}{$\begin{array}{l}\text { Flesh apparent fractal dimension by Richardson plot } \\
\text { (FFDR) }\end{array}$} \\
\hline EC1 & 1 & $\begin{array}{l}f f d r- \\
1.1\end{array}$ & 2.4 & 5.88 & Encore 10 & 0.010 & $77153-2204911$ & 0.13 \\
\hline & 3 & $\begin{array}{l}f f d r- \\
3.1\end{array}$ & 81.8 & 10.11 & Encore 10 & 0.017 & $16211979-28040750$ & 0.27 \\
\hline
\end{tabular}

a pfi, placenta firmness; sfi, skin firmness; ffi, flesh firmness; fci, flesh crispness index; ffdr, flesh apparent fractal dimension based on Richardson plot; ffdk, flesh apparent fractal dimension based on box-counting (Kolmogorov's dimension).

${ }^{b} s f i-1.2^{E}$ is contributed by 'Encore $10^{\prime}$ inbred line, sfi-3. $1^{A}$ is contributed by 'Atar' inbred line, sfi- $1.2^{C}$ and $s f i-3.1^{C}$ are contributed by CS-PMR1.

${ }^{\mathrm{c}} R^{2}$ is the proportion of phenotypic variance explained by the QTL. 


\begin{tabular}{|c|c|c|c|c|c|c|c|c|}
\hline Trait & Chr. & $\mathrm{QTL}^{\mathrm{ab}}$ & $\begin{array}{l}\text { Position } \\
\text { (cM) }\end{array}$ & LOD peak & $\begin{array}{l}\text { Contributing } \\
\text { parent }\end{array}$ & $\begin{array}{l}\text { Additive } \\
\text { effect }\end{array}$ & $\begin{array}{l}\text { Estimated range within } \\
\text { 'Chinese Long' v3 genome }\end{array}$ & $R^{2 c}$ \\
\hline & 5 & $\begin{array}{l}f f d r- \\
5.1\end{array}$ & 79.6 & 5.17 & Encore 10 & 0.011 & $17657831-24443816$ & 0.12 \\
\hline \multirow[t]{3}{*}{ EC2 } & 1 & $\begin{array}{l}f f d r- \\
1.1\end{array}$ & 9.7 & 9.03 & Encore 10 & 0.014 & $62696-4118470$ & 0.17 \\
\hline & 2 & $\begin{array}{l}f f d r- \\
2.1\end{array}$ & 48.6 & 4.03 & Encore 10 & 0.008 & $13530264-14476230$ & 0.07 \\
\hline & 3 & $\begin{array}{l}f f d r- \\
3.1\end{array}$ & 55.3 & 6.30 & Encore 10 & 0.015 & $17838480-29875711$ & 0.12 \\
\hline \multirow[t]{6}{*}{ EA } & 1 & $\begin{array}{l}f f d r- \\
1.2\end{array}$ & 37.5 & 3.53 & Atar & -0.008 & $9538226-10199104$ & 0.06 \\
\hline & 2 & $\begin{array}{l}f f d r- \\
2.2\end{array}$ & 73.8 & 3.76 & Atar & -0.009 & $17477386-20408580$ & 0.08 \\
\hline & 3 & $\begin{array}{l}f f d r- \\
3.1\end{array}$ & 70.2 & 10.38 & Encore 10 & 0.016 & $16175273-29445989$ & 0.21 \\
\hline & 5 & $\begin{array}{l}f f d r- \\
5.1\end{array}$ & 67.9 & 5.76 & Encore 10 & 0.011 & $20648581-27048928$ & 0.11 \\
\hline & 6 & $\begin{array}{l}f f d r- \\
6.1\end{array}$ & 62.7 & 6.80 & Encore 10 & 0.010 & $13642268-22008822$ & 0.13 \\
\hline & 6 & $\begin{array}{l}f f d r- \\
6.2\end{array}$ & 90.3 & 3.61 & Encore 10 & 0.006 & $24693006-25831572$ & 0.06 \\
\hline \multicolumn{9}{|c|}{ Flesh apparent fractal dimension by box-counting (Kolmogorov's dimension) (FFDK) } \\
\hline \multirow[t]{3}{*}{ EC1 } & 1 & $\begin{array}{l}f f d k- \\
1.1\end{array}$ & 7.5 & 9.29 & Encore 10 & 0.015 & $77153-5251504$ & 0.18 \\
\hline & 3 & $\begin{array}{l}f f d k- \\
3.1\end{array}$ & 84.0 & 7.77 & Encore 10 & 0.016 & $16298404-28040750$ & 0.22 \\
\hline & 5 & $\begin{array}{l}f f d k- \\
5.1\end{array}$ & 22.6 & 4.94 & Encore 10 & 0.012 & $2754900-5011135$ & 0.13 \\
\hline \multirow[t]{2}{*}{ EC2 } & 1 & $\begin{array}{l}f f d k- \\
1.1\end{array}$ & 1.6 & 10.94 & Encore 10 & 0.018 & $62696-2502042$ & 0.19 \\
\hline & 3 & $\begin{array}{l}f f d k- \\
3.1\end{array}$ & 58.0 & 11.43 & Encore 10 & 0.019 & $17838480-29875711$ & 0.21 \\
\hline \multirow[t]{4}{*}{ EA } & 2 & $\begin{array}{l}f f d k- \\
2.1\end{array}$ & 59.2 & 4.43 & Atar & -0.010 & $17148765-20408580$ & 0.08 \\
\hline & 3 & $\begin{array}{l}f f d k- \\
3.1\end{array}$ & 70.2 & 10.44 & Encore 10 & 0.017 & $16175273-29445989$ & 0.20 \\
\hline & 5 & $\begin{array}{l}f f d k- \\
5.2\end{array}$ & 67.9 & 5.18 & Encore 10 & 0.011 & $21011617-27048928$ & 0.09 \\
\hline & 6 & $\begin{array}{l}f f d k- \\
6.1\end{array}$ & 62.7 & 3.99 & Encore 10 & 0.007 & $16965857-21093120$ & 0.07 \\
\hline
\end{tabular}

a pfi, placenta firmness; sfi, skin firmness; ffi, flesh firmness; fci, flesh crispness index; ffdr, flesh apparent fractal dimension based on Richardson plot; ffdk, flesh apparent fractal dimension based on box-counting (Kolmogorov's dimension).

${ }^{b} s f i-1.2^{E}$ is contributed by 'Encore $10^{\prime}$ inbred line, sfi-3. $1^{A}$ is contributed by 'Atar' inbred line, $s f i-1.2^{C}$ and $s f i-3.1^{C}$ are contributed by CS-PMR1.

${ }^{\mathrm{c}} R^{2}$ is the proportion of phenotypic variance explained by the QTL. 


\begin{tabular}{|c|c|c|c|c|c|c|c|c|}
\hline Trait & Chr. & $\mathrm{QTL}^{\mathrm{ab}}$ & $\begin{array}{l}\text { Position } \\
\text { (cM) }\end{array}$ & LOD peak & $\begin{array}{l}\text { Contributing } \\
\text { parent }\end{array}$ & $\begin{array}{l}\text { Additive } \\
\text { effect }\end{array}$ & $\begin{array}{l}\text { Estimated range within } \\
\text { 'Chinese Long' v3 genome }\end{array}$ & $R^{2 c}$ \\
\hline & 6 & $\begin{array}{l}f f d k- \\
6.2\end{array}$ & 90.3 & 4.15 & Encore 10 & 0.008 & $24693006-26281957$ & 0.07 \\
\hline \multicolumn{9}{|c|}{$\begin{array}{l}\text { a pfi, placenta firmness; sfi, skin firmness; ffi, flesh firmness; fci, flesh crispness index; ffdr, flesh apparent fractal dimension } \\
\text { based on Richardson plot; } f f d k \text {, flesh apparent fractal dimension based on box-counting (Kolmogorov's dimension). }\end{array}$} \\
\hline \multicolumn{9}{|c|}{$\begin{array}{l}\text { b } s f i-1.2^{E} \text { is contributed by 'Encore } 10^{\prime} \text { inbred line, sfi-3.1 } 7^{A} \text { is contributed by 'Atar' inbred line, sfi-1.2 } 2^{C} \text { and } s f i-3.1^{C} \text { are contributed } \\
\text { by CS-PMR } 1 \text {. }\end{array}$} \\
\hline
\end{tabular}

All QTLs are named with the trait abbreviation followed by the LG number and the sequential number of the QTL for this trait $(1,2,3$, or 4) along the chromosome (Table 1). The QTLs for SFI on Chrs. 1 and 3 were detected in the same region among the three populations, but the contributing parents were different. These QTL names were appended with the initial letter of their parent name in superscript. Some major QTLs for different texture traits were detected in almost the same region (Fig. 4; Table 1): pfi-1.1, sfi-1.1, ffi-1.1, fci-1.1, ffdr-1.1, and ffdk-1.1 on one part of Chr. $1 ;$ sfi-1.2 $2^{E}$ and ffi-1.2 on another part of Chr. 1; pfi-2.1 and sfi-2.1 on Chr. 2; fci3.1, ffdr-3.1, and ffdk-3.1 on Chr. 3; pfi-6.1, sfi-6.1, and fci-6.1 on Chr. 6.

\section{Discussion}

In cucumber breeding, fruit texture traits are primary targets for improving fruit quality. Breeders have hitherto assessed fruit texture traits by sensory evaluation. Genotypic effects and heritability of fruit firmness have been investigated in crossing experiments, and it has been suggested that firmness is controlled genetically (Peterson et al. 1978; Kanno and Kamimura 1980; Yoshioka et al. 2009, 2010). However, few genetic studies of fruit crispness in cucumber have been performed (Yoshioka et al. 2009, 2010). Evaluating fruit texture traits quantitatively by using mechanical measurement in segregating populations allows their genetic analysis. As all texture traits of our three populations of $F_{2}$ progeny were distributed widely, these traits are controlled quantitatively (Fig. 2). Many fruit appearance traits, such as length, diameter, and L/D, are controlled by QTLs (Yuan et al. 2008a, b; Bo et al. 2015; Weng et al. 2015; Pan et al. 2017; Shimomura et al. 2017; Gao et al. 2020; Pan et al. 2020; Wang et al. 2020a, b). Our results indicate that fruit texture traits also are controlled by QTLs.

Linkage maps of three $F_{2}$ populations were constructed by using ddRAD-Seq analysis and compared (Fig. 3). The order of common markers is almost identical among the three populations and the cucumber reference genome (Li et al. 2019). Polymorphic loci were detected more or less evenly throughout each LG in EC1 and EC2. However, in EA, there were relatively large gaps, of $>10 \mathrm{cM}$, between markers. This result suggests that the 'Encore 10' inbred line and the 'Atar' inbred line have similar structures in part of the genome because they were both derived from current $F_{1}$ cultivars, and thus polymorphic sites cannot be detected in these parts.

Fifty-six QTLs for firmness (PFI, SFI, FFI) and crispness-related scores (FCl, FFDR, FFDK) were detected (Fig. 4; Table 1). Some of the QTLs with large effect for PFI and SFI were detected in the same regions in EC1 and EC2 but in different regions in EA. The flesh is the most important part because it accounts for most of the cucumber fruit. Composite interval mapping detected 8 QTLs for FFI, 10 for $\mathrm{FCl}, 8$ for FFDR, and 7 for FFDK. Those on Chr. 1 were detected in all populations (Fig. 4). However, their effects differed between EC and EA: In EC1 and EC2, ffi-1.2 (in different locations from those for crispness-related scores), fci-1.1, ffdr-1.1, and ffdk1.1 had large effects. In EA, on the other hand, ffi-1.1 had a large effect, but $f c i-1.1$ and $f f d r-1.2$ had very small effects. Although there were some overlapping regions, crispness and firmness appear to be controlled by different mechanisms. Furthermore, QTLs for all crispness-related scores on Chr. 3 were detected in all populations, and some had high LOD scores (Fig. 4). Those on Chr. 6 were detected in both EC1 and EA (Fig. 4). Yoshioka et al. (2009) reported that fruit texture parameters differed significantly among cultivation periods. However, these QTLs, especially those on Chr. 3, were detected in multiple populations and cultivation periods, indicating their importance in controlling flesh crispness traits.

There are no reports of QTLs for firmness and crispness in cucumber, but some reports describe QTL analysis and gene identification of fruit texture traits in other species. Several QTLs and candidate genes for fruit firmness are reported in melon

Page $10 / 18$ 
(Moreno et al. 2008), grape (Carreño et al. 2014; Correa et al. 2016), and tomato (Chapman et al. 2012; Yang et al. 2016). In apple, $M d-P G 1$, in the polygalacturonase gene family, was associated with loss of fruit firmness and crispness during storage (Costa et al. 2010; Longhi et al. 2013; Moriya et al. 2017; Poles et al. 2020). Md-PG1 is related to cell wall structure and its modification. In the cucumber reference genome, a few polygalacturonaselike genes are located within the estimated QTL range for flesh crispnessrelated scores detected in Chr. 3. However, flesh crispness in cucumber still diffed among cultivars before harvesting (Shimomura et al. 2016). The results indicate that a variety of genetic mechanisms may govern fruit crispness.

CS-PMR1 can be used as breeding material because of its strong resistance to powdery mildew and downy mildew (Sakata et al. 2006; Fukino et al. 2013; Yoshioka et al. 2014). Some large-effect QTLs for resistance to these diseases are located near the QTLs detected here. In particular, the QTLs with large effects on SFI, FFI, and crispness-related scores on Chr. 1 identified here (Fig. 4) are located near some genes for resistance to powdery mildew (pm1.1 and pm1.2) and downy mildew ( $d m 1.2$ and $d m$ 1.3) (Fukino et al. 2013; Yoshioka et al. 2014). Furthermore, QTLs for fruit length, diameter, and L/D ratio were detected on Chrs. 1, 2, and 6 in different populations (Yuan et al. 2008a, b; Bo et al. 2015; Weng et al. 2015; Pan et al. 2017; Shimomura et al. 2017; Gao et al. 2020; Wang et al. 2020a, b). The locations of QTLs for fruit texture, morphological traits, and disease resistance on these chromosomes are highly complex. In developing new cultivars with introgressed traits, breeders repeat backcrossing and selecting over many years. However, the apparent linkage between fruit texture traits and resistance would reduce breeding efficiency in gene pyramiding. Our result will contribute to the development of new DNA markers tightly linked to QTLs for fruit texture traits that can be used in breeding.

Fruit firmness and crispness differ greatly among cultivars around the world, and consumers have different preferences. In cucumber, fruit texture is considered to be the most important deliciousness factor in Japan, because cucumber has little distinctive taste-related characteristics, such as sweetness, as are found in other fruit crops and vegetables. For example, flesh crispness scores were significantly higher in Japanese $F_{1}$ hybrid cultivars than in other cultivars such as the Beit Alpha type and the European greenhouse type (Yoshioka et al. 2009; Sakata et al. 2011; Shimomura et al. 2016). Therefore, fruit crispness has been an important target in cucumber breeding. The newly identified QTLs for fruit firmness and crispness will allow us to develop DNA markers with the traits. In particular, the QTLs on Chr. 3, where QTL for flesh crispness-related scores were detected among all populations, would be crucial to selecting for high crispness. We anticipate that our results will advance the development of informative DNA markers for fruit texture traits, and of new cucumber cultivars with desired fruit qualities.

\section{Declarations}

\section{Ethics approval and consent to participate}

Not applicable.

\section{Consent for publication}

Not applicable.

\section{Availability of data and materials}

Data that support the findings of this study are available from the corresponding author on reasonable request.

\section{Conflicts of interest}

The authors declare that they have no conflict of interest.

\section{Funding}

This work was supported by a Grant-in-Aid for Young Scientists (A), JSPS KAKENHI Grant Number JP26712004. 


\section{Authors' contributions}

All authors contributed to the study conception and design. Material preparation, data collection and analysis were performed by $Y Y$ and KS. All authors discussed and guided all steps of the experiments, and contributed to the preparation of the final version of the paper.

\section{Acknowledgments}

We thank Dr. Motoki Shimizu of Iwate Biotechnology Research Center for coaching in NGS data analysis, and Dr. Kenta Shirasawa of Kazusa DNA Research Institute for instruction in ddRAD-Seq analysis. We also thank F. Hori, S. Masuji, D. Yamashita, Y. Wazaki, M. Shindo, M. Wakabayashi, Y. Taki, A. Suzuki, C. Yamada, A. Tsunoda, and M. Sano of the Institute of Vegetable and Floriculture Science, NARO, for technical assistance.

\section{References}

[1] Bo K, Ma Z, Chen J, Weng Y (2015) Molecular mapping reveals structural rearrangements and quantitative trait loci underlying traits with local adaptation in semi-wild Xishuangbanna cucumber (Cucumis sativus L. var. xishuangbannanesis Qi et Yuan). Theor Appl Genet 128:25-39. https://doi.org/10.1007/s00122-014-2410-z

[2] Breene WM, Da Vis DW, Chou HE (1972) Texture profile analysis of cucumbers. J Food Sci 37:113-117. https://doi.org/10.1111/j.1365-2621.1972.tb03398.x

[3] Carreño I, Cabezas JA, Martínez-Mora C, Arroyo-García R, Cenis JL, Martínez-Zapater JM, Carreño J, Ruiz-García L (2014) Quantitative genetic analysis of berry firmness in table grape (Vitis vinifera L.). Tree Genet Genomes 11:818.

https://doi.org/10.1007/s11295-014-0818-x

[4] Cavagnaro P, Senalik D, Yang L, Simon P, Harkins T, Kodira C, Huang S, Weng Y (2010) Genome-wide characterization of simple sequence repeats in cucumber (Cucumis sativus L.). BMC Genom 11:569. https://doi.org/10.1186/1471-2164-11-569

[5] Chapman NH, Bonnet J, Grivet L, Lynn J, Graham N, Smith R, Sun G, Walley PG, Poole M, Causse M, King GJ, Baxter C, Seymour GB (2012) High-resolution mapping of a fruit firmness-related quantitative trait locus in tomato reveals epistatic interactions associated with a complex combinatorial locus. Plant Physiol 159:1644-1657. https://doi.org/10.1104/pp.112.200634

[6] Correa J, Mamani M, Muñoz-Espinoza C, González-Agüero M, Defilippi BG, Campos-Vargas R, Pinto M, Hinrichsen P (2016) New stable QTLs for berry firmness in table grapes. Am J Enol Vitic 67:212-217. https://doi.org/10.5344/ajev.2015.15049

[7] Costa F, Peace CP, Stella S, Serra S, Musacchi S, Bazzani M, Sansavini S, Van de Weg WE (2010) QTL dynamics for fruit firmness and softening around an ethylene-dependent polygalacturonase gene in apple (Malus $\times$ domestica Borkh.). J Exp Bot 61:30293039. https://doi.org/10.1093/jxb/erq130

[8] Dan H, Okuhara K, Kohyama K (2003) Discrimination of cucumber cultivars using a multiple-point sheet sensor to measure biting force. J Sci Food Agric 83:1320-1326. https://doi.org/10.1002/jsfa.1543

[9] Davey JW, Hohenlohe PA, Etter PD, Boone JQ, Catchen JM, Blaxter ML (2011) Genome-wide genetic marker discovery and genotyping using next-generation sequencing. Nat Rev Genet 12:499-510. https://doi.org/10.1038/nrg3012

[10] Fazio G, Staub JE, Stevens MR (2003) Genetic mapping and QTL analysis of horticultural traits in cucumber (Cucumis sativus L.) using recombinant inbred lines. Theor Appl Genet 107:864-874. https://doi.org/10.1007/s00122-003-1277-1

[11] Fukino N, Ohara T, Monforte AJ, Sugiyama M, Sakata Y, Kunihisa M, Matsumoto S (2008) Identification of QTLs for resistance to powdery mildew and SSR markers diagnostic for powdery mildew resistance genes in melon (Cucumis melo L.). Theor Appl Genet 118:165-175. https://doi.org/10.1007/s00122-008-0885-1

Page $12 / 18$ 
[12] Fukino N, Yoshioka Y, Sugiyama M, Sakata Y, Matsumoto S (2013) Identification and validation of powdery mildew (Podosphaera xanthii)-resistant loci in recombinant inbred lines of cucumber (Cucumis sativus L.). Mol Breed 32:267-277. https://doi.org/10.1007/s11032-013-9867-3

[13] Gao Z, Zhang H, Cao C, Han J, Li H, Ren Z (2020) QTL mapping for cucumber fruit size and shape with populations from long and round fruited inbred lines. Hortic Plant J 6:132-144. https://doi.org/10.1016/j.hpj.2020.04.004

[14] Goffinet MC (1977) Some anatomical considerations in the study of cucumber fruit texture. J Am Soc Hortic Sci 102:474-478

[15] Horie H, Ito H, Ippoushi K, Azuma K, Igarashi I (2004) Evaluation of the physical quality of the mesocarp of cucumber fruit. Hort Res (Jpn) 3:425-428

[16] Iwata H, Ninomiya S (2006) AntMap: constructing genetic linkage maps using an ant colony optimization algorithm. Breed Sci 56:371-377. https://doi.org/10.1270/jsbbs.56.371

[17] Jeon IJ, Breene WM, Munson ST (1973) Texture of cucumbers: correlation of instrumental and sensory measurements. J Food Sci 38:334-337. https://doi.org/10.1111/j.1365-2621.1973.tb01419.x

[18] Jones ID (1954) Varietal differences in cucumbers for pickling. Food Tech 8:415-418

[19] Kanno T, Kamimura S (1978) Measuring textural characteristics and varietal differences of skin toughness and flesh firmness in the cucumber fruit. Bull Veg Ornam Crop Res Stn Ser B 2:25-42

[20] Kanno T, Kamimura S (1980) Inheritance study of skin toughness and flesh firmness of cucumber fruit in $F_{1}$ generation. Bull Veg Ornam Crop Res Stn Ser B 3:29-38

[21] Kennard WC, Havey MJ (1995) Quantitative trait analysis of fruit quality in cucumber: QTL detection, confirmation, and comparison with mating-design variation. Theor Appl Genet 91:53-61. https://doi.org/10.1007/BF00220858

[22] Kohyama K, Nagata A, Tamaki Y, Sakurai N (2009) Comparison of human-bite and instrument puncture tests of cucumber texture. Postharvest Biol Technol 52:243-246. https://doi.org/10.1016/j.postharvbio.2008.12.001

[23] Kohyama K, Kato-Nagata A, Shimada H, Kazami Y, Hayakawa F (2013) Texture of sliced cucumbers measured by subjective human-bite and objective instrumental tests. J Texture Stud 44:1-11. https://doi.org/10.1111/j.1745-4603.2012.00360.x

[24] Li H (2011) A statistical framework for SNP calling, mutation discovery, association mapping and population genetical parameter estimation from sequencing data. Bioinformatics 27:2978-2993. https://doi.org/10.1093/bioinformatics/btr509

[25] Li H (2013) Aligning sequence reads, clone sequences and assembly contigs with BWA-MEM. arXiv:13033997v2 [q-bioGN]

[26] Li Q, Li H, Huang W, Xu Y, Zhou Q, Wang S, Ruan J, Huang S, Zhang Z (2019) A chromosome-scale genome assembly of cucumber (Cucumis sativus L.). GigaScience 8: 1-10. https://doi.org/10.1093/gigascience/giz072

[27] Lo C, Chain PS (2014) Rapid evaluation and quality control of next generation sequencing data with FaQCs. BMC Bioinform 15: 366. https://doi.org/10.1186/s12859-014-0366-2

[28] Longhi S, Hamblin MT, Trainotti L, Peace CP, Velasco R, Costa F (2013) A candidate gene based approach validates Md-PG1 as the main responsible for a QTL impacting fruit texture in apple (Malus × domestica Borkh). BMC Plant Biol 13:37.

https://doi.org/10.1186/1471-2229-13-37

[29] Moreno E, Obando JM, Dos-Santos N, Fernández-Trujillo JP, Monforte AJ, Garcia-Mas J (2008) Candidate genes and QTLs for fruit ripening and softening in melon. Theor Appl Genet 116:589-602. https://doi.org/10.1007/s00122-007-0694-y

[30] Morishita M, Sugiyama K, Saito T, Sakata Y (2003) Powdery mildew resistance in cucumber. Jpn Agric Res Q 37:7-14. https://doi.org/10.6090/jarq.37.7 
[31] Moriya S, Kunihisa M, Okada K, Iwanami H, Iwata H, Minamikawa M, Katayose Y, Matsumoto T, Mori S, Sasaki H, Matsumoto T, Nishitani C, Terakami S, Yamamoto T, Abe K (2017) Identification of QTLs for flesh mealiness in Apple (Malus $\times$ domestica Borkh.). Hort J 86:159-170. https://doi.org/10.2503/hortj.MI-156

[32] Pan Y, Liang X, Gao M, Liu H, Meng H, Weng Y, Cheng Z (2017) Round fruit shape in WI7239 cucumber is controlled by two interacting quantitative trait loci with one putatively encoding a tomato SUN homolog. Theor Appl Genet 130:573-586.

https://doi.org/10.1007/s00122-016-2836-6

[33] Pan Y, Wang Y, McGregor C, Liu S, Luan F, Gao M, Weng Y (2020) Genetic architecture of fruit size and shape variation in cucurbits: a comparative perspective. Theor Appl Genet 133:1-21. https://doi.org/10.1007/s00122-019-03481-3

[34] Peleg M (1997) Measures of line jaggedness and their use in foods textural evaluation. Critical Rev Food Sci Nutrition 37:491518. https://doi.org/10.1080/10408399709527787

[35] Peterson RK, Davis DW, Stucker RE, Breene WM (1978) Inheritance of firmness in raw cucumber (Cucumis sativus L.) fruit. Euphytica 27:233-240. https://doi.org/10.1007/BF00039140

[36] Poles L et al. (2020) Role of fruit flesh cell morphology and MdPG1 allelotype in influencing juiciness and texture properties in apple. Postharvest Biol Technol 164:111161. https://doi.org/10.1016/j.postharvbio.2020.111161

[37] Ren Y, Zhang Z, Liu J, Staub JE, Han Y, Cheng Z, Li X, Lu J, Miao H, Kang H, Xie B, Gu X, Wang X, Du Y, Jin W, Huang S (2009) An integrated genetic and cytogenetic map of the cucumber genome. PLoS One 4:e5795.

https://doi.org/10.1371/journal.pone.0005795

[38] Roudaut G, Dacremont C, Vallès Pàmies B, Colas B, Le Meste M (2002) Crispness: a critical review on sensory and material science approaches. Trends Food Sci Technol 13:217-227. https://doi.org/10.1016/S0924-2244(02)00139-5

[39] Russ JC (1994) Fractal Surfaces. Plenum Press, Now York, pp 27-57

[40] Sakata Y, Kubo N, Morishita M, Kitadani E, Sugiyama M, Hirai M (2006) QTL analysis of powdery mildew resistance in cucumber (Cucumis sativus L.). Theor Appl Genet 112:243-250. https://doi.org/10.1007/s00122-005-0121-1

[41] Sakata Y, Horie H, Yoshioka Y, Sugiyama M (2011) Fruit textures of Beit Alpha, Greenhouse, Japanese, Pickling, and Slicer-type cucumbers. J Jpn Soc Hort Sci 80:420-425. https://doi.org/10.2503/jjshs1.80.420

[42] Sakurai N, Iwatani S, Terasaki S, Yamamoto R (2005) Texture evaluation of cucumber by a new acoustic vibration method. J Jpn Soc Hort Sci 74:31-35. https://doi.org/10.2503/jjshs.74.31

[43] Serquen FC, Bacher J, Staub JE (1997) Mapping and QTL analysis of horticultural traits in a narrow cross in cucumber (Cucumis sativus L.) using random-amplified polymorphic DNA markers. Mol Breed 3:257-268.

https://doi.org/10.1023/A:1009689002015

[44] Shimomura K, Horie H, Sugiyama M, Kawazu Y, Yoshioka Y (2016) Quantitative evaluation of cucumber fruit texture and shape traits reveals extensive diversity and differentiation. Sci Hortic 199:133-141. https://doi.org/10.1016/j.scienta.2015.12.033

[45] Shimomura K, Fukino N, Sugiyama M, Kawazu Y, Sakata Y, Yoshioka Y (2017) Quantitative trait locus analysis of cucumber fruit morphological traits based on image analysis. Euphytica 213:138. https://doi.org/10.1007/s10681-017-1926-0

[46] Shirasawa K, Hirakawa H, Isobe S (2016) Analytical workflow of double-digest restriction site-associated DNA sequencing based on empirical and in silico optimization in tomato. DNA Res 23:145-153. https://doi.org/10.1093/dnares/dsw004

[47] Shimizu T, Yano K (2011) A post-labeling method for multiplexed and multicolored genotyping analysis of SSR, indel and SNP markers in single tube with bar-coded split tag (BStag). BMC Res Notes 4:161. https://doi.org/10.1186/1756-0500-4-161

[48] Suojala-Ahlfors T (2005) Fruit firmness of pickling cucumber cultivars. Horttechnology 15:777-781.

https://doi.org/10.21273/HORTTECH.15.4.0777

Page 14/18 
[49] Thompson RL, Fleming HP, Hamann DD, Monroe RJ (1982) Method for determination of firmness in cucumber slices. J Texture Stud 13:311-324. https://doi.org/10.1111/j.1745-4603.1982.tb00886.x

[50] Wang S, Basten C, Zeng Z (2007) Windows QTL Cartographer 2.5. Department of Statistics, North Carolina State University, Raleigh, NC. http://statgen.ncsu.edu/qtlcart/WQTLCart.htm

[51] Wang X, Li H, Gao Z, Wang L, Ren Z (2020a) Localization of quantitative trait loci for cucumber fruit shape by a population of chromosome segment substitution lines. Sci Rep 10:11030. https://doi.org/10.1038/s41598-020-68312-8

[52] Wang Y, Bo K, Gu X, Pan J, Li Y, Chen J, Wen C, Ren Z, Ren H, Chen X, Grumet R, Weng Y (2020b) Molecularly tagged genes and quantitative trait loci in cucumber with recommendations for QTL nomenclature. Hort Res 7:3. https://doi.org/10.1038/s41438-0190226-3

[53] Wei Q, Wang Y, Qin X, Zhang Y, Zhang Z, Wang J, Li J, Lou Q, Chen J (2014) An SNP-based saturated genetic map and QTL analysis of fruit-related traits in cucumber using specific-length amplified fragment (SLAF) sequencing. BMC Genom 15:1158. https://doi.org/10.1186/1471-2164-15-1158

[54] Weng Y, Colle M, Wang Y, Yang L, Rubinstein M, Sherman A, Ophir R, Grumet R (2015) QTL mapping in multiple populations and development stages reveals dynamic quantitative trait loci for fruit size in cucumbers of different market classes. Theor Appl Genet 128:1747-1763. https://doi.org/10.1007/s00122-015-2544-7

[55] Yang S, Yu Q, Wang B, Yang T, Li N, Tang Y, Aisimutuola P, Wang Q, Xu J, Gao J (2016) Identification of QTLs for red fruit firmness using the wild tomato species Solanum pennellii LA716 introgression lines. Plant Breed 135:728-734.

https://doi.org/10.1111/pbr.12423

[56] Yoshioka Y, Horie H, Sugiyama M, Sakata Y (2009) Quantifying cucumber fruit crispness by mechanical measurement. Breed Sci 59:139-147. https://doi.org/10.1270/jsbbs.59.139

[57] Yoshioka Y, Sugiyama M, Sakata Y (2010) Combining ability analysis of fruit texture traits in cucumber by mechanical measurement. Breed Sci 60:65-70. https://doi.org/10.1270/jsbbs.60.65

[58] Yoshioka Y, Sakata Y, Sugiyama M, Fukino N (2014) Identification of quantitative trait loci for downy mildew resistance in cucumber (Cucumis sativus L.). Euphytica 198:265-276. https://doi.org/10.1007/s10681-014-1102-8

[59] Yuan XJ, Li XZ, Pan JS, Wang G, Jiang S, Li XH, Deng SL, He HL, Si MX, Lai L, Wu AZ, Zhu LH, Cai R (2008a) Genetic linkage map construction and location of QTLs for fruit-related traits in cucumber. Plant Breed 127:180-188.

https://doi.org/10.1111/j.1439-0523.2007.01426.x

[60] Yuan XJ, Pan JS, Cai R, Guan Y, Liu LZ, Zhang WW, Li Z, He HL, Zhang C, Si LT, Zhu LH (2008b) Genetic mapping and QTL analysis of fruit and flower related traits in cucumber (Cucumis sativus L.) using recombinant inbred lines. Euphytica 164:473-491. https://doi.org/10.1007/s10681-008-9722-5

\section{Figures}




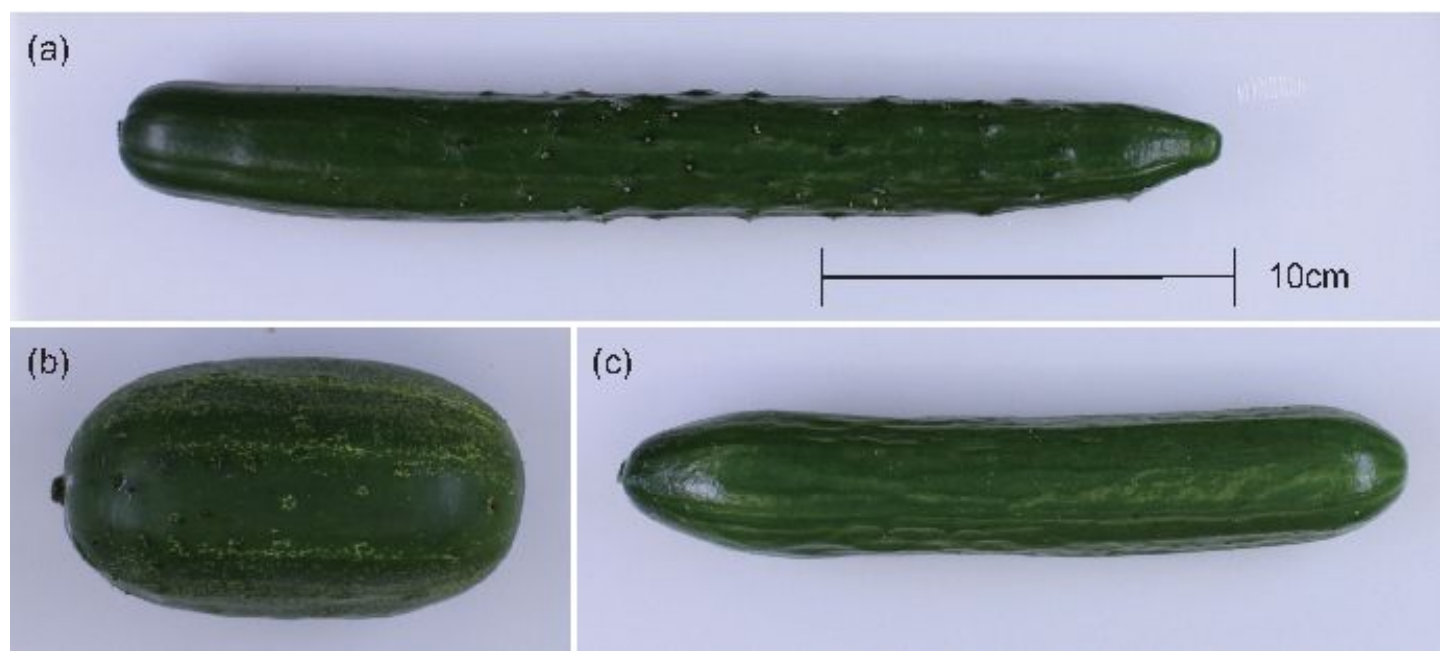

\section{Figure 1}

Fruit of the parental lines at $100 \mathrm{~g}$ weight: a high-crisp inbred line 'Encore 10', b low-crisp line CS-PMR1, c low-crisp inbred line 'Atar'.
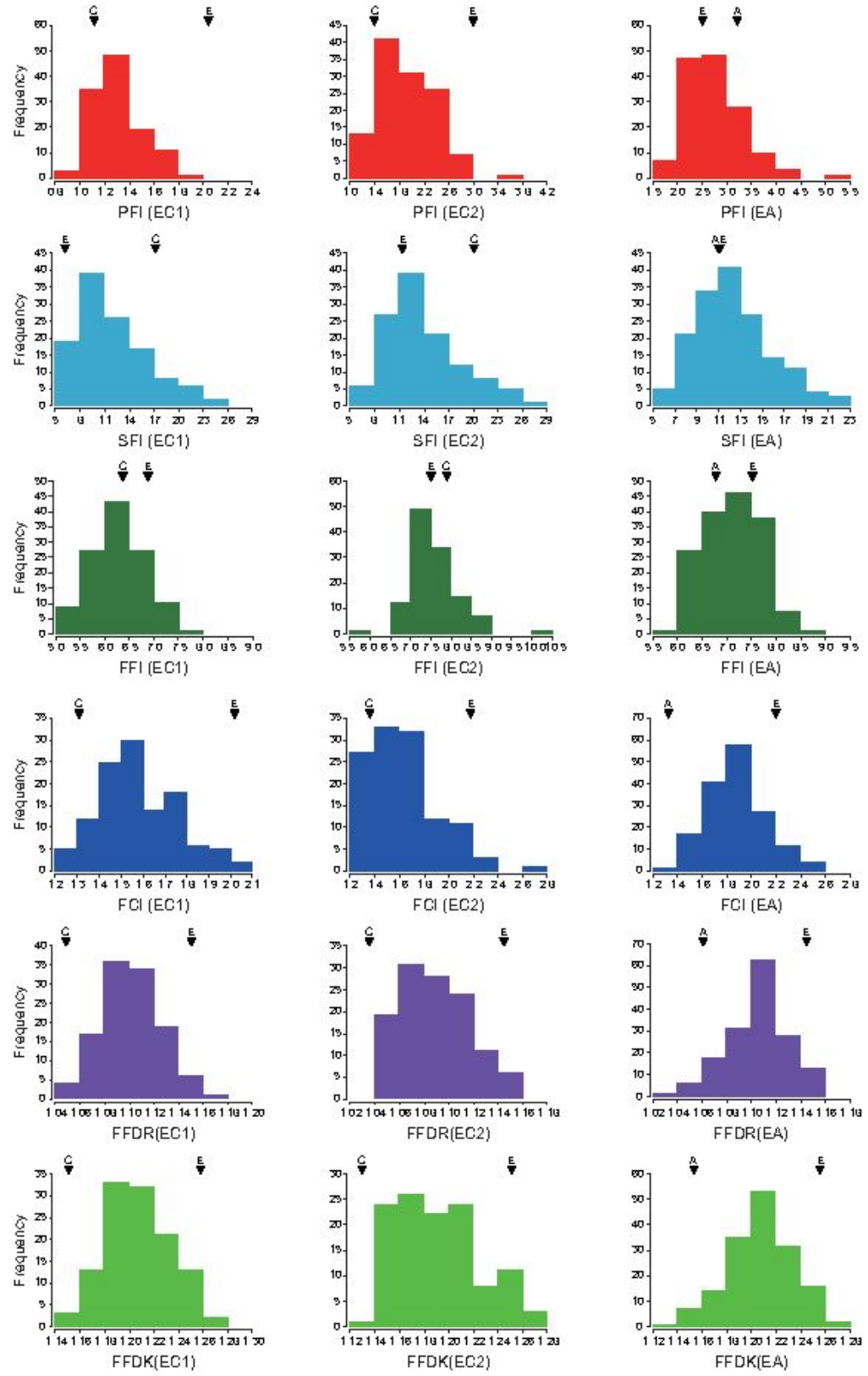
Figure 2

Frequency distributions of fruit texture traits in F2 populations derived from 'Encore 10' ("E") × CS-PMR1 ("C ${ }^{\prime}$ ) crosses (EC1, EC2) and $\mathrm{E} \times$ 'Atar' ("A") cross (EA). PFI, placenta firmness; SFI, skin firmness; FFI, flesh firmness; FCl, flesh crispness index; FFDR, flesh apparent fractal dimensions based on Richardson plot; FFDK, flesh apparent fractal dimension based on box-counting (Kolmogorov's dimension).
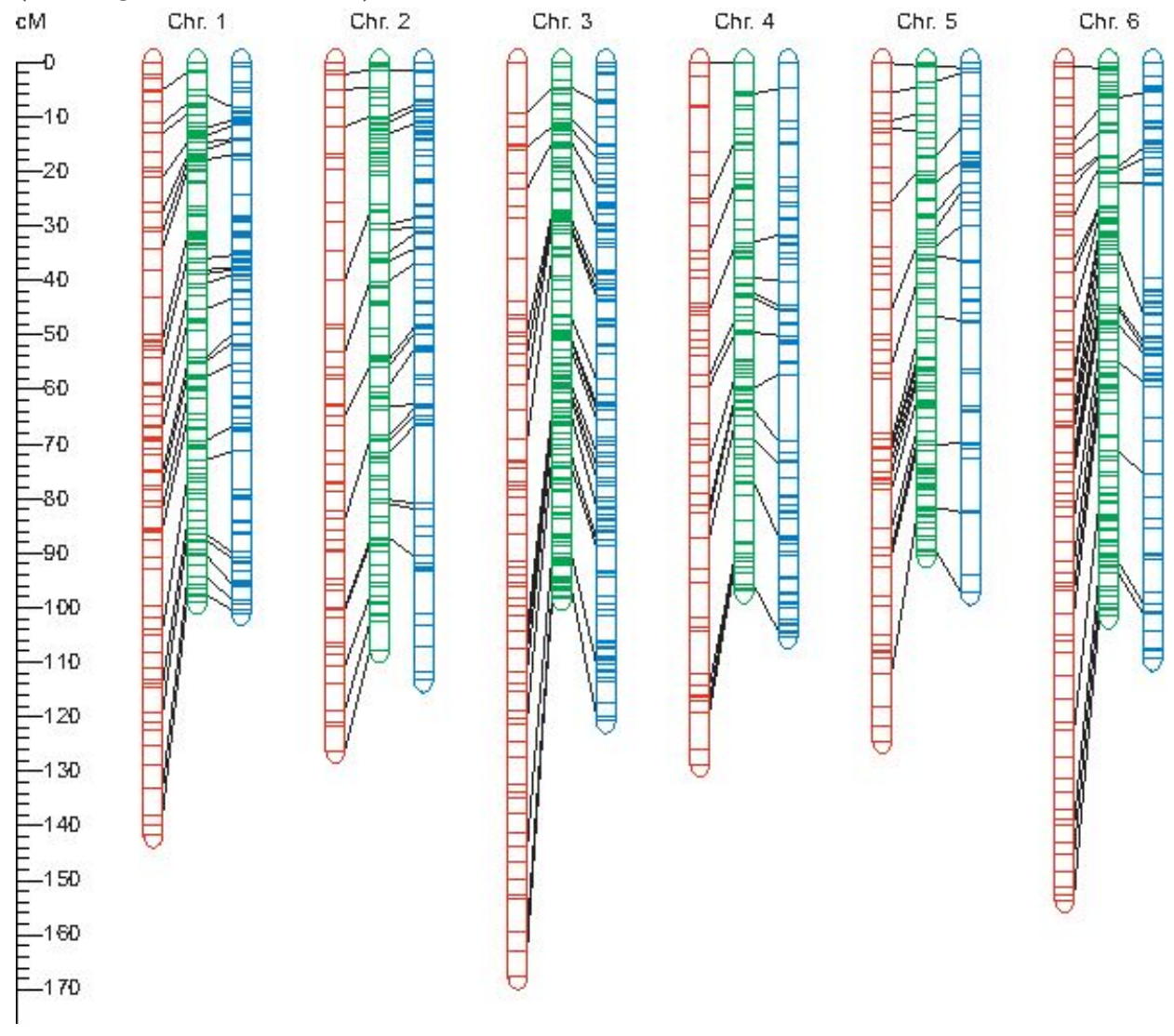

Chr. 7

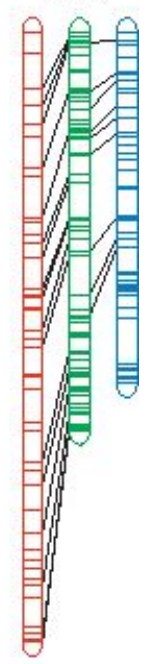

\section{Figure 3}

Linkage map of 'Encore 10' $\times$ CS-PMR1 crosses (EC1, EC2) and 'Encore 10' $\times$ 'Atar' cross (EA). Linkage groups were assigned to cucumber reference genome chromosomes ('Chinese Long v3'). Left position is EC1, center is EC2, and right is EA in each linkage group. Ladder steps in each linkage group indicate loci; black lines between linkage groups indicate common loci among (or between) populations.
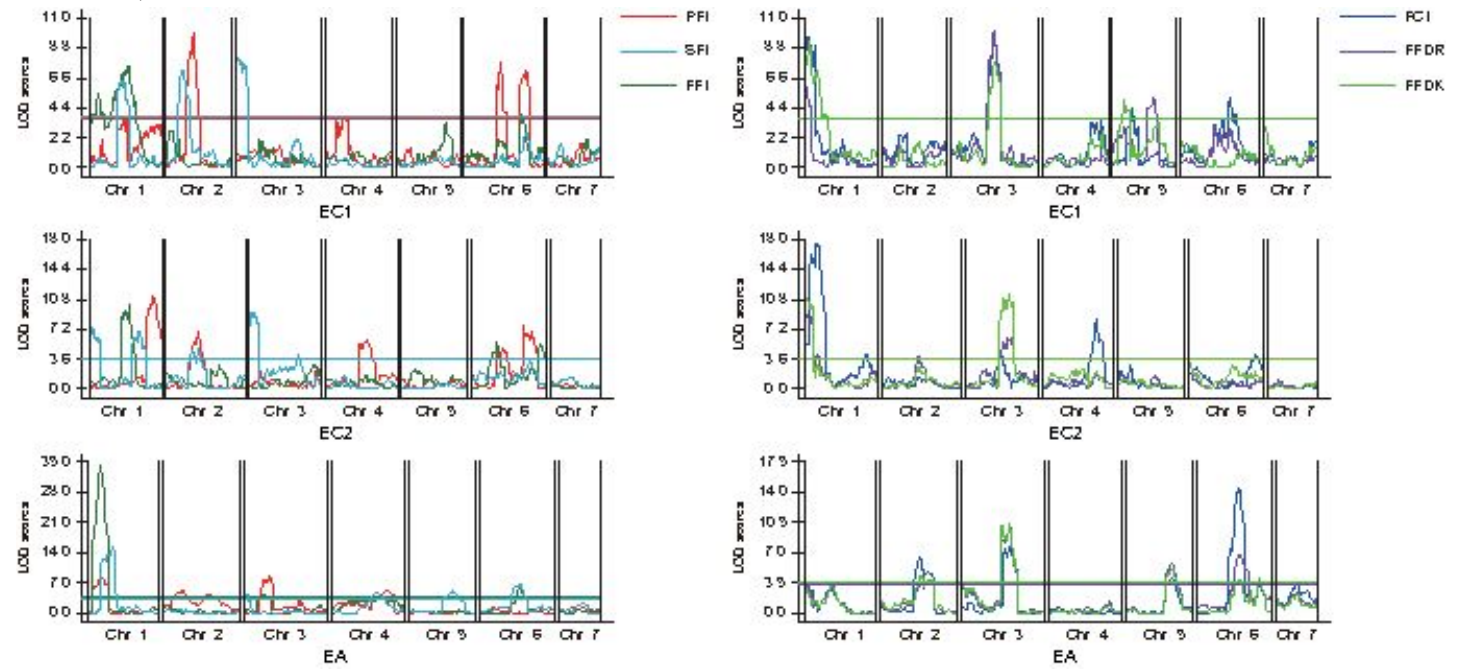

Figure 4 
Positions and LOD scores of QTLs associated with fruit texture traits in three F2 populations. Horizontal rules represent LOD thresholds at the 0.05 level of significance. PFI, placenta firmness; SFI, skin firmness; $\mathrm{FFI}$, flesh firmness; $\mathrm{FCl}$, flesh crispness index; FFDR, flesh apparent fractal dimensions based on Richardson plot; FFDK, flesh apparent fractal dimension based on box-counting (Kolmogorov's dimension). 